\title{
HOMOTOPY TYPES OF THE DELETED PRODUCT OF UNIONS OF TWO SIMPLEXES
}

\author{
W. T. WHITLEY
}

\begin{abstract}
If $X$ is a space, let $X^{*}=X \times X-D$, where $D$ is the diagonal. If $f$ is a map on $X$ to a space $Y$, let $X_{f}^{*}=\left\{(x, y) \in X^{*} \mid f(x) \neq\right.$ $f(y)\}$. In this paper we continue our investigation, begun in [6], of the homotopy types of $X^{*}$ and $X_{f}^{*}$, and of a question due to Brahana $\left[1\right.$, p. 236], as to when the homotopy types of $X^{*}$ and $X_{f}^{*}$ are the same. If $X$ is the union of two nondisjoint simplexes, and if $f$ is a simplicial map on $X$, we are able, using results and techniques developed in [6], to express the homotopy types of $X^{*}$ and $X_{f}^{*}$ in terms of spheres, and then to determine when the homotopy types of these spaces are the same.
\end{abstract}

1. Introduction and notation. If $X$ is a finite polyhedron and $f$ is a simplicial map on $X$, let

$$
P\left(X^{*}\right)=\bigcup\{r \times s \mid r \text { and } s \text { are simplexes in } X \text { with } r \cap s=\varnothing\},
$$

and let $P\left(X_{f}^{*}\right)=\bigcup\{r \times s \mid r$ and $s$ are simplexes in $X$ with $f(r) \cap f(s)=\varnothing\}$. In [2, pp. 351-352], Hu has shown that $P\left(X^{*}\right)$ and $X^{*}$ are homotopically equivalent, and in $\left[3\right.$, p. 183] Patty has observed that $P\left(X_{f}^{*}\right)$ and $X_{f}^{*}$ are homotopically equivalent.

The symbol $\left\langle v_{0}, \cdots, v_{n}\right\rangle$ will denote the $n$-simplex with vertices $v_{0}, \cdots, v_{n}$. We let $S^{n}=\left\{x \in E^{n+1}|| x \mid=1\right\}$. If $X$ and $Y$ are spaces, " $X \simeq Y$ " will mean that $X$ and $Y$ are homotopically equivalent.

2. The results. For the remainder of this paper, we let $X=A \cup B$, where $A=\left\langle v_{0}, \cdots, v_{k}, v_{k+1}, \cdots, v_{n}\right\rangle$ and $B=\left\langle v_{0}, \cdots, v_{k}, w_{k+1}, \cdots, w_{m}\right\rangle$ are $n$ and $m$-simplexes, respectively, such that $A \cap B=\left\langle v_{0}, \cdots, v_{k}\right\rangle$ is a nonempty $k$-simplex, $k<n, k<m$.

THEOREM 1. Let $f: A \rightarrow Y$ be a simplicial map, $Y$ a polyhedron. Then $P\left(A_{f}^{*}\right) \simeq P\left(f(A)^{*}\right) \simeq S^{q-1}$, where $q=\operatorname{dim} f(A)$.

Proof. There is a face $w$ of $A$, say $w=\left\langle v_{0}, \cdots, v_{q}\right\rangle$, such that $f(w)=$ $f(A)$ and $f \mid w$ is a homeomorphism. Hence, $P\left(w_{f}^{*}\right)=P\left(w^{*}\right) \simeq P\left(f(w)^{*}\right)=$ $P\left(f(A)^{*}\right)$.

Presented to the Society, May 18, 1970; received by the editors October 2, 1970.

AMS 1970 subject classifications. Primary 55D10; Secondary 55E05.

(c) American Mathematical Suciety $19 / 2$ 
Let $g: A \rightarrow w$ be the simplicial map defined by $g(v)=w^{\prime} \cap f^{-1} f(v)$ for each vertex $v$ in $A$. Then $g^{2}=g$, and $g$ takes $A$ into $A$. Hence, $P\left(A_{g}^{*}\right) \simeq P\left(g(A)^{*}\right)=$ $P\left(w^{*}\right)$ (Corollary 2.1 of $[6]$ ).

It is straightforward to show that $P\left(A_{g}^{*}\right)=P\left(A_{f}^{*}\right)$, so we have $P\left(A_{f}^{*}\right) \simeq$ $P\left(f(A)^{*}\right)$. Since $f(A)$ is a simplex of dimension $q$, the remainder of the theorem follows from Corollary 1 of [4].

Let $f$ be a simplicial map on $X$ to a polyhedron $Y$. Let

$D_{1}=\bigcup\{r \times s \mid r$ is a face of $A, s$ is a face of $B$, and $f(r) \cap f(s)=\varnothing\}$, and let $D_{2}=\bigcup\{r \times s \mid r$ is a face of $B, s$ is a face of $A$, and $f(r) \cap f(s)=\varnothing\}$. Note that $P\left(X_{f}^{*}\right)=P\left(A_{f}^{*}\right) \cup P\left(B_{f}^{*}\right) \cup D_{1} \cup D_{2}$.

LEMMA 1. If $f(B)$ is not contained in $f(A)$, then $D_{1}, D_{2}, D_{1} \cap P\left(B_{f}^{*}\right)$ and $D_{2} \cap P\left(B_{f}^{*}\right)$, are all contractible.

Proof. Let $r$ and $s$ be simplexes in $X$ with $r \times s \subset D_{1}$. There exists a vertex $v_{B} \in B$ such that $f\left(v_{B}\right) \notin f(A)$. We may assume $v_{B} \in s$. Then if $g$ is the linear map defined on $D_{1}$ by $g(r \times s)=r \times\left\{v_{B}\right\}$, it is clear that $D_{1} \simeq g\left(D_{1}\right)$. Now if $r \times\left\{v_{B}\right\}$ is a cell in $g\left(D_{1}\right)$, we may assume $v_{0} \in r$. Then it is clear that $g\left(D_{1}\right) \simeq\left\{\left(v_{0}, v_{B}\right)\right\}$. Therefore, $D_{1}$ is contractible. Since $D_{1}$ and $D_{2}$ are homeomorphic, $D_{2}$ is also contractible.

Now observe that if $r \times s$ is a cell in $D_{1} \cap P\left(B_{f}^{*}\right), r$ is a face of $A \cap B$, and $s$ is a face of $B$, and since $v_{0} \in A \cap B$, we may contract $D_{1} \cap P\left(B_{f}^{*}\right)$ to $\left(v_{0}, v_{B}\right)$ by the same argument as above. Similarly, $D_{2} \cap P\left(B_{f}^{*}\right)$ is contractible.

The proof of the following lemma is straightforward and hence is left to the reader.

Lemma 2. If $f(A) \neq f(A \cap B)$, then $D_{1} \cap P\left(A_{f}^{*}\right)$ and $D_{2} \cap P\left(A_{f}^{*}\right)$ are contractible.

LEMMA 3. If $f(B)$ is not a subset of $f(A)$, then $P\left(B_{f}^{*}\right) \simeq P\left(B_{f}^{*}\right) \cup D_{1} \cup D_{2}$ If, in addition, $f(A) \neq f(A \cap B)$, then $P\left(A_{f}^{*}\right) \simeq P\left(A_{f}^{*}\right) \cup D_{1} \cup D_{2}$.

Proof. By Lemma 1, $P\left(B_{f}^{*}\right) \simeq P\left(B_{f}^{*}\right) \cup D_{1}$. Clearly, $D_{1} \cap D_{2} \subset P\left(B_{f}^{*}\right) \cap$ $D_{2}$, so $\left(P\left(B_{f}^{*}\right) \cup D_{1}\right) \cap D_{2}=P\left(B_{f}^{*}\right) \cap D_{2}$, which is contractible by Lemma 1 . Then since $D_{2}$ is contractible, $P\left(B_{f}^{*}\right) \simeq P\left(B_{f}^{*}\right) \cup D_{1} \simeq P\left(B_{f}^{*}\right) \cup D_{1} \cup D_{2}$. Similarly, using Lemma 2, we have $P\left(A_{f}^{*}\right) \simeq P\left(A_{f}^{*}\right) \cup D_{1} \cup D_{2}$.

Lemma 4. If there is a vertex $v_{-A} \in A$ such that $f\left(v_{-A}\right) \notin f(A \cap B)$, then $\left(D_{1} \cup D_{2}\right) \cap P\left(A_{f}^{*}\right) \simeq P\left(\left\langle v_{0}, \cdots, v_{k}, v_{A}\right\rangle_{f}^{*}\right)$.

Proof. The proof is the same as the proof of Lemma 4 of [6].

THEOREM 2. Let $f$ be a simplicial map on $X$. Suppose there exist vertices $v_{A} \in A$ and $v_{B} \in B$ such that $f\left(v_{A}\right) \notin f(A \cap B)$ and $f\left(v_{B}\right) \notin f(A)$. If $n=k+1$, then $P\left(X_{f}^{*}\right) \simeq S^{r}$, where $r+1=\operatorname{dim} f(B)$; if $m=k+1$, then $P\left(X_{f}^{*}\right) \simeq S^{q}$, where 
$q+1=\operatorname{dim}_{1} f(A) ;$ if $m, n>k+1$, then $P\left(X_{f}^{*}\right) \simeq S^{q} \cup S^{r}$, where $S^{q} \cap S^{r} \simeq S^{t}$, $t+1=\operatorname{dim} f\left(\left\langle v_{0}, \cdots, v_{k}, v_{A}\right\rangle\right)$

Procf. If $n=k+1$, then $P\left(A_{f}^{*}\right) \subset P\left(B_{f}^{*}\right) \cup D_{1} \cup D_{2}$. Then $P\left(X_{f}^{*}\right)=$ $P\left(B_{f}^{*}\right) \cup D_{1} \cup D_{2}$. But $P\left(B_{f}^{*}\right) \cup D_{1} \cup D_{2} \simeq P\left(B_{f}^{*}\right) \simeq S^{r}$ from Theorem 1 and Lemma 3. Hence, $P\left(X_{f}^{*}\right) \simeq S^{r}$. Similarly, if $m=k+1, P\left(X_{f}^{*}\right) \simeq S^{q}$.

Now assume $m, n>k+1$. By Theorem $1, P\left(A_{f}^{*}\right) \simeq S^{q}$ and $P\left(B_{f}^{*}\right) \simeq S^{r}$. Then by Lemma 3, $P\left(B_{f}^{*}\right) \cup D_{1} \cup D_{2} \simeq S^{r}$. Then

$$
\begin{array}{rlrl}
\left(P\left(B_{f}^{*}\right) \cup D_{1} \cup\right. & \left.D_{2}\right) \cap P\left(A_{f}^{*}\right) \\
& =\left[P\left(A_{f}^{*}\right) \cap P\left(B_{f}^{*}\right)\right] \cup\left[\left(D_{1} \cup D_{2}\right) \cap P\left(A_{f}^{*}\right)\right] \\
& =\left[P\left((A \cap B)_{f}^{*}\right)\right] \cup\left[\left(D_{1} \cup D_{2}\right) \cap P\left(A_{f}^{*}\right)\right] \\
& =\left(D_{1} \cup D_{2}\right) \cap P\left(A_{f}^{*}\right) & \\
& \simeq P\left(\left\langle v_{0}, \cdots, v_{k}, v_{A}\right\rangle_{f}^{*}\right) & & \text { by Lemma } 4 \\
& \simeq S^{t} & & \text { by Theorem } 1 .
\end{array}
$$

This proves the theorem.

COROLlaRY 2.1. If $n=k=1, P\left(X^{*}\right) \simeq S^{m-1}$; if $m=k+1, P\left(X^{*}\right) \simeq S^{n-1}$; if $m, n>k+1, P\left(X^{*}\right) \simeq S^{m-1} \cup S^{n-1}$, with $S^{m-1} \cap S^{n-1} \simeq S^{k}$.

Proof. Let $f$ be the identity map in Theorem 2 .

We should note that the results of Corollary 2.1 for the cases $m=k+1$ or $n=k+1$ are special cases of Theorem 6 of [5].

Note further that Corollary 2.1 allows us to compute the homology groups of $P\left(X^{*}\right)$ easily.

We now need to consider the cases where both of the conditions (1) $f(A) \neq f(A \cap B)$ and $f(B) \ddagger f(A)$ and (2) $f(B) \neq f(A \cap B)$ and $f(A) \ddagger f(B)$ fail simultaneously. There are three such possibilities: (i) $f(A)=f(A \cap B)$, (ii) $f(B)=f(A \cap B)$, (iii) $f(A)=f(B) \neq f(A \cap B)$.

THEOREM 3. If $f(A)=f(A \cap B)$, then $P\left(X_{f}^{*}\right) \simeq P\left(B_{f}^{*}\right)$; if $f(B)=f(A \cap B)$, then $P\left(X_{f}^{*}\right) \simeq P\left(A_{f}^{*}\right)$.

Proof. Suppose $f(A)=f(A \cap B)$. There is a face $u$ of $B$ such that $f(u)=$ $f(B)=f(X), f(u \cap A)=f(A \cap B)=f(A)$, and $f \mid u$ is a homeomorphism. Let $g: X \rightarrow X$ be the simplicial map defined by $g(v)=u \cap f^{-1} f(v)$. Then by Corollary 2.1 of $[6], P\left(X_{g}^{*}\right) \simeq P\left(g(X)^{*}\right)=P\left(g(B)^{*}\right) \simeq P\left(B_{g}^{*}\right)$. It is straightforward to show that $P\left(X_{g}^{*}\right)=P\left(X_{f}^{*}\right)$ and $P\left(B_{g}^{*}\right)=P\left(B_{f}^{*}\right)$ since $f=f g$, and $f \mid u$ is one-to-one. Hence, $P\left(X_{f}^{*}\right) \simeq P\left(B_{f}^{*}\right)$.

Similarly, if $f(B)=f(A \cap B)$, then $P\left(X_{f}^{*}\right) \simeq P\left(A_{f}^{*}\right)$.

Corollary 3.1. If $f(A)=f(A \cap B)$, then $P\left(X_{f}^{*}\right) \simeq S^{r}$, where $r+1=$ $\operatorname{dim} f(B)$; if $f(B)=f(A \cap B)$, then $P\left(X_{f}^{*}\right) \simeq S^{q}$, where $q+1=\operatorname{dim} f(A)$.

Proc)F. This is a direct consequence of Theorem 1 and Theorem 3. 
Now suppose $f(A)=f(B) \neq f(A \cap B)$. Then we may choose faces $r_{1}=\left\langle u_{0}, \cdots, u_{p}, u_{p+1}, \cdots, u_{q}\right\rangle \subset A$ and $r_{2}=\left\langle u_{0}, \cdots, u_{p}, t_{p+1}, \cdots, t_{q}\right\rangle=B$ such that $f\left(r_{1}\right)=f(A), f\left(r_{2}\right)=f(B), r_{1} \cap r_{2}=\left\langle u_{0}, \cdots, u_{p}\right\rangle$ with $f\left(r_{1} \cap r_{2}\right)=$ $f(A \cap B)$, and $f \mid r_{i}$ is a homeomorphism, $i=1,2$.

Lemma 5. Let $W=r_{1} \cup r_{2}$. Then $P\left(W_{f}^{*}\right) \simeq P\left(X_{f}^{*}\right)$.

Proof. The proof is the same as the proof of Lemma 7 of [6].

We may now assume $f(W)=r_{1}$ since $f(W)=f\left(r_{1}\right)$ and $f \mid r_{1}$ is a homeomorphism.

LEMMA 6. If $p=0$ and $q=p+1, P\left(W_{f}^{*}\right)$ has the homotopy type of four points. If $p \geqq 1$ and $q=p+1$, the $(q-1)$ st homology group of $P\left(W_{f}^{*}\right)$ is isomorphic to the direct sum of three copies of the integers. If $q>p+1$, the $(q-1)$ st homology group of $P\left(W_{f}^{*}\right)$ is isomorphic to a group which contains the direct sum of three copies of the integers as a proper subgroup.

Proof. If $p=0$ and $q=p+1$, the result is trivial. Otherwise, since $f \mid r_{i}$ is a homeomorphism, $i=1,2$, and $f(W)=f\left(r_{1}\right)=r_{1}$, the result follows directly from Case I of the proof of Lemma 9 of [6] since $P\left(r_{1 f}^{*}\right) \simeq P\left(r_{2 f}^{*}\right) \simeq$ $S^{q-1}$ by Theorem 1 .

Theorem 4. If $f(A)=f(B) \neq f(A \cap B)$, then $P\left(X^{*}\right) \neq P\left(X_{f}^{*}\right)$.

Proof. If $m=k+1, P\left(X^{*}\right) \simeq S^{n-1}$ by Corollary 3.1. But by Lemma 6, $P\left(X_{f}^{*}\right) \neq S^{n-1}$. Similarly, if $n=k+1, P\left(X^{*}\right) \neq P\left(X_{f}^{*}\right)$.

Suppose $m, n>k+1$. Then by Lemma 6 , some nonzero homology group of $P\left(X_{f}^{*}\right)$ contains a direct summand of three copies of the integers as a subgroup. By Corollary 2.1 , some nonzero homology group of $P\left(X^{*}\right)$ contains a subgroup isomorphic to a direct summand of three copies of the integers if and only if $m=n=k+2$, and in this case, the $(m-1)$ st homology group of $P\left(X^{*}\right)$ is isomorphic to the direct sum of three copies of the integers. But by Lemma 6 , the $(m-1)$ st homology group of $P\left(X_{f}^{*}\right)$ is isomorphic to the direct sum of three copies of the integers if and only if $\operatorname{dim} f(X)=m=1+\operatorname{dim} f(A \cap B)$, which is impossible if $m=k+2$.

Corollary 4.1. Suppose $f$ is any simplicial map on $X$. Then $P\left(X^{*}\right) \simeq$ $P\left(X_{f}^{*}\right)$ if and only if (1) $f(A)=f(A \cap B), \operatorname{dim} f(X)=m$, and $n=k+1$; (2) $f(B)=f(A \cap B), \operatorname{dim} f(X)=n$, and $m=k+1$; (3) $f(B) \nsubseteq f(A), f(A) \neq$ $f(A \cap B), n=k+1$, and $\operatorname{dim} f(B)=m$; (4) $f(A) \ddagger f(B), f(B)=f(A \cap B)$, $m=k+1$, and $\operatorname{dim} f(A)=n ;(5) f(B) \ddagger f(A), f(A)=f(A \cap B), m, n>k+1$, $\operatorname{dim} f(B)=m, \operatorname{dim} f(A)=n$, or $\operatorname{dim} f(B)=n, \operatorname{dim} f(A)=m ;$ or $(6) f(A) \ddagger$ $f(B), f(B) \neq f(A \cap B), m, n>k+1, \operatorname{dim} f(B)=m, \operatorname{dim} f(A)=n, \operatorname{or} \operatorname{dim} f(B)$ $=n, \operatorname{dim} f(A)=m$.

Proof. This follows directly from the preceding results. 


\section{BIBLIOGRAPHY}

1. T. R. Brahana, On a class of isotopy invariants, Topology of 3-Manifolds and Related Topics (Proc. The Univ. of Georgia Inst., 1961), Prentice-Hall, Englewood Cliffs, N.J., 1962, pp. 235-237. MR 25 \#5514.

2. S. T. Hu, Isotopy invariants of topological spaces, Proc. Roy. Soc. 255 (1970), 331366.

3. C. W. Patty, Isotopy invariants of trees, Duke Math. J. 31 (1964), 183-197. MR 28 \#2548.

4. - Polyhedra whose deleted products have the homotopy type of the n-sphere, Duke Math. J. 36 (1969), 233-236. MR 40 \#2076.

5. - Deleted products with homotopy types of spheres, Trans. Amer. Math. Soc. 147 (1970), 223-240. MR 40 \#6558.

6. W. T. Whitley, Deleted products of spaces which are unions of two simplexes, Trans. Amer. Math. Soc. 157 (1971), 99-111.

Department of Mathematics, Marshall University, Huntington, West VIRGINIA 25701 